\title{
Persistent laminations from Seifert surfaces
}

\author{
MARK BRITTENHAM ${ }^{1}$ \\ University of North Texas
}

\begin{abstract}
We show how an incompressible Seifert surface $F$ for a knot $K$ in $S^{3}$ can be used to create an essential lamination $\mathcal{L}_{F}$ in the complement of each of an infinite class of knots associated to $F$. This lamination is persistent for these knots; it remains essential under all non-trivial Dehn fillings of the knot complement. This implies a very strong form of Property $\mathrm{P}$ for each of these knots.
\end{abstract}

INTRODUCTION

Essential laminations have found many uses in 3-manifold topology, principally in understanding Dehn fillings on 3-manifolds. For example, Delman [De] showed that 2-bridge knots have Property $\mathrm{P}$, by finding essential laminations in their complements which remain essential under all non-trivial Dehn fillings. Such laminations are called persistent, and the knots are called persistently laminar. Naimi [Na1],[Na2], independently, proved the same result using different methods. In fact, since each of these Dehn filled manifolds contains an essential lamination, each has universal cover $\mathbb{R}^{3}[\mathrm{GO}]$, a property which we have chosen to call strong Property $\mathrm{P}$ for the knot. Since reducible manifolds do not have universal cover $\mathbb{R}^{3}$, strong Property P implies the cabling conjecture for the knot, as well.

More generally, Delman and Roberts [DR], using a mixture of the above methods, have proved strong Property $\mathrm{P}$ for non-torus alternating knots. Recently, $\mathrm{Wu}[\mathrm{Wu}]$ proved strong Property $\mathrm{P}$ for most algebraic knots, again using essential laminations.

In this paper we describe a process for generating knots with strong Property $\mathrm{P}$, by building essential laminations in the complement of a knot using an incompressible Seifert surface for some other knot. The knots which we succeed in doing this for are usually not alternating, and are probably not algebraic, since they do not seem to decompose into rational tangles.

This construction was also discovered, around the same time, by Ulrich Oertel (unpublished).

Key words and phrases. essential lamination, Seifert surface, Property P.

${ }^{1}$ Research supported in part by NSF grants \# DMS-9400651 and DMS-9708411 
The Motivating EXAMPLE

The reader is referred to $[R]$ for background in knot theory and Dehn surgery. Many of the basic concepts about essential laminations and branched surfaces may be found in [GO].

The genesis of the constructions we give here is an example due to Ulrich Oertel [Oe1], which was analyzed, from the point of view of tangles, in [Br1]. There a branched surface $B$ was constructed in the complement of a certain tangle $T_{0}$, in the 3-ball $B^{3} ; B$ carried a lamination $\mathcal{L}$ which was essential in the complement of any knot obtained by tangle sum with $T_{0}$ (see Figure 1) Further, the lamination remained essential after non-trivial Dehn surgery along any of the knots so constructed.

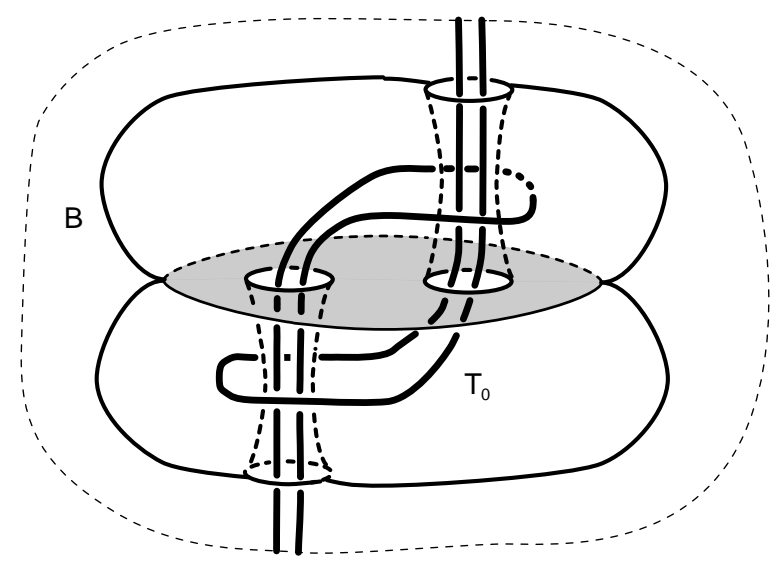

Figure 1

This lamination and branched surface are, as we shall see, the simplest of a long list of laminations and branched surfaces, that can be associated to any incompressible Seifert surface for a knot. They come, in fact, from applying our construction to a 2-disk spanning the unknot. We start with this example, in order to motivate the general construction.
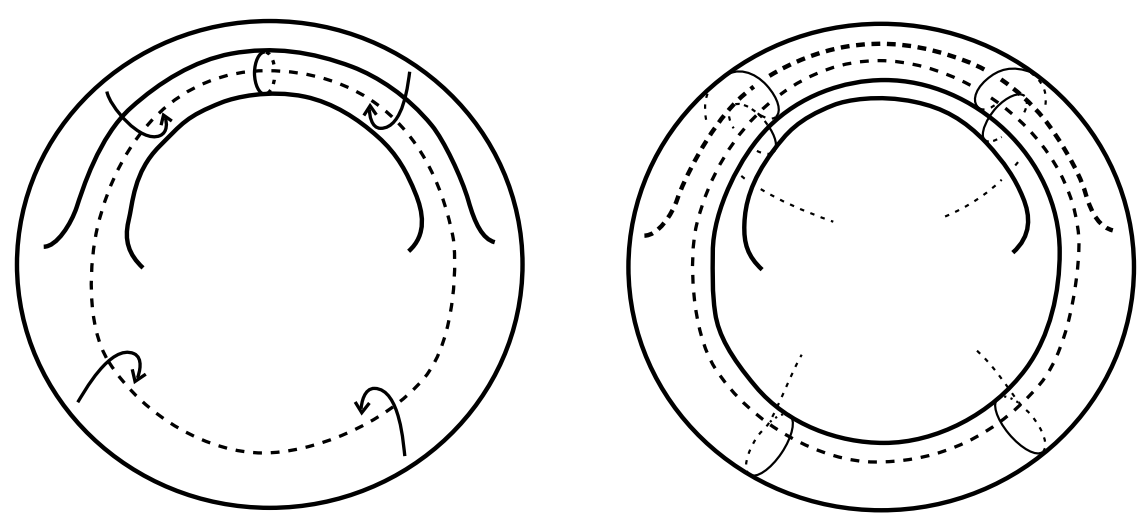

Figure 2

We can obtain one view of Oertel's branched surface $B$ by starting with a 2-disk 
$D$ spanning the unknot, gluing a tube to it, and then gluing the boundary of the resulting punctured torus onto the punctured torus, in so doing creating a branch curve which runs over the tube (see Figure 2). The reader is invited to verify that the resulting branched surface is isotopic to the one in Figure 1, although this is not really central to what follows. If we then string a pair of arcs through both the tube we added to $D$ and the tube created when we glued the branched surface together (Figure 3), and then complete the pair of arcs to a knot $K$ in any way that avoids the branched surface and the two tubes (i.e., the two compressing disks for the tubes), then it is not hard to see [Br1] that the branched surface $B$ is essential in the complement of the knot. Furthermore, since the boundaries of two compressing disks of $\partial_{h} N(B)$ in $S^{3}$ are not isotopic in $\partial_{h} N(B)$ - they live on different components - and each compressing disk intersects our knot exactly once, it follows, primarily from Menasco's criterion [Me], that our branched surface remains essential after any non-trivial Dehn surgery along the knot $K$ [Br1]. The knot we have chosen to picture here is in fact the twist knot $6_{1}$, as the reader can verify.

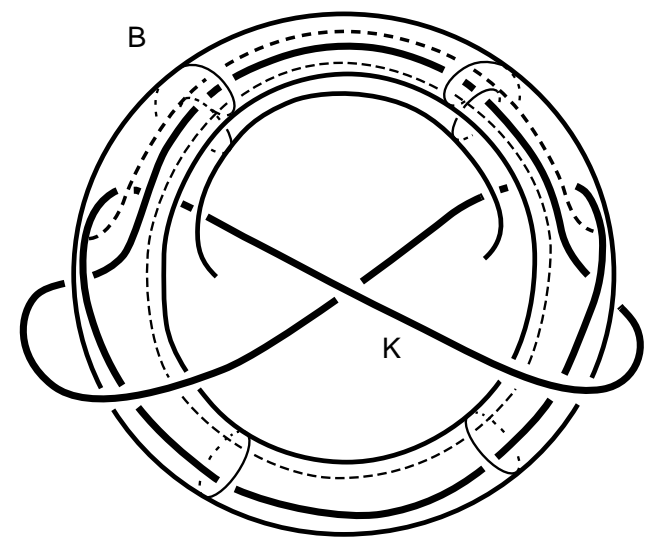

Figure 3

The fact that we will exploit to find our more general construction is that the construction of $B$ did not really use the topology of the disk $D$ (or lack of it); it was constructed from $D$ by alterations taking place only in a neighborhood of the boundary of $D$. We can therefore apply the same construction to any surface $F$ with boundary in the 3 -sphere, i.e., to any Seifert surface $F$ for a knot $K=\partial F$ in the 3 -sphere. (The construction can be applied to non-orientable surfaces as well, although conditions which guarantee that the resulting laminations are persistent are somewhat harder to quantify; see [Br2].) What we shall see is that if this process is applied to an incompressible Seifert surface for a knot, the lamination we create is persistent for any knot that we construct in the same manner as above.

\section{The CONSTRUCTIONS}

The construction is completely analogous to the one pictured in Figure 2; we simply forget that we can see the entire disk $D$ and focus on a neighborhood of its boundary. Given a Seifert surface $F$ for a knot $K$, a neighborhood of its boundary is an annulus; if we attach a tube to this annulus, running parallel to half of the 
knot $K$, and then glue the boundary of $F$ (i.e., the knot $K$ ) to a curve running over the tube, and otherwise following the other half of the knot $K$, we get a branched surface $B_{F}$; see Figure 4 . There are actually two ways to do this gluing, to get a branched surface; we choose the one which makes $B_{F}$ transversely orientable, as in the figure. The horizontal boundary of $B_{F}$ then splits into a positive part, which we will call $\partial_{+}$, and a negative part $\partial_{-}$. This branched surface has a single branch curve $\gamma$, which has no self-intersections, and so it is easy to build a ' $\beta$-measured' lamination $\mathcal{L}$ carried by $B_{F}$ with full support (see [Br1] or [Ro]).

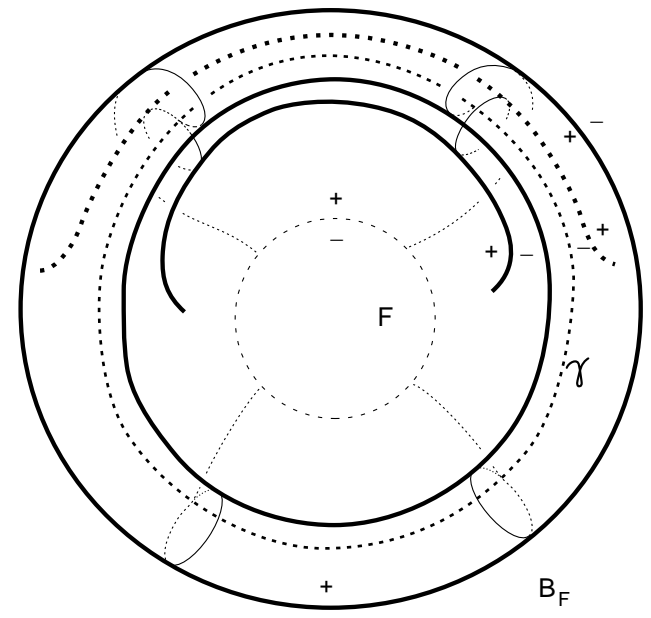

Figure 4

To understand what will happen next, we need a good picture of what the complement of a neighborhood of $B_{F}, M_{B}=S^{3} \backslash \operatorname{int} N\left(B_{F}\right)$, looks like. Let $M_{K}=$ $S^{3} \backslash \operatorname{int} N(K)$. The idea is to think of the modifications described above as taking place in the solid torus neighborhood $N(K)$ of $K$ in $S^{3}$, so that $M_{B} \cap M_{K}=$ $M_{K} \backslash \operatorname{int} N(F)=M_{F} . M_{F}$ can be thought of as a sutured manifold, with an annular suture $A$ separating two copies of $F$, which we will call $F_{+}$and $F_{-}$. The Seifert surface $F$ is incompressible precisely when $F_{+}$and $F_{-}$are incompressible in $M_{F}$.

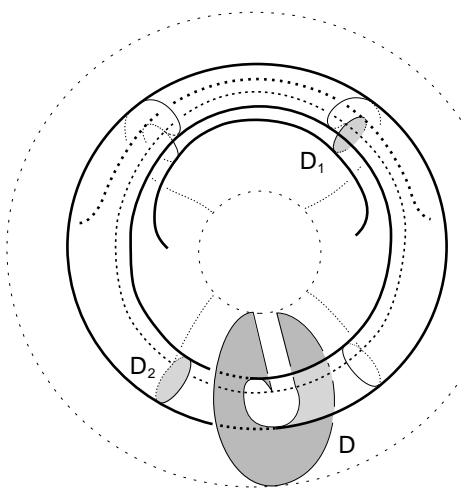

Figure 5

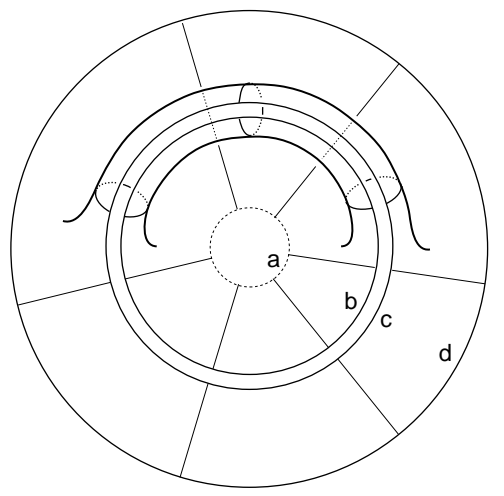

Figure 6

To understand $M_{B}$, it therefore suffices to understand what $N(K) \backslash \operatorname{int} N(B)=$ $M_{B} \cap N(K)=N$ looks like. This is a sutured manifold, with two sutures, one 
$\left(A_{1}\right)$ being the vertical boundary $\partial_{v} N\left(B_{F}\right)$, and the other $\left(A_{2}\right)$ being the annulus $\partial \mathrm{N}(\mathrm{K}) \backslash \operatorname{intN}(\mathrm{B})$ (see Figure 5). By inspection, the complement of the two sutures in $\partial N$ is a pair of twice-punctured tori; they are each built from $\left(B_{F} \cap N(K)\right) \backslash \gamma$, which is a 4-punctured sphere (Figure 6 ), by gluing pairs of boundary circles together $(b$ to $c$ for the one from $\partial_{+}, c$ to $d$ for the one from $\left.\partial_{-}\right)$. We will call these two $\partial$-components $B_{+}$and $B_{-}$. Each of these punctured tori is compressible in $N$, via the meridian disks $D_{1}, D_{2}$ in our two tubes (Figure 5).

Compressing $\partial N$ along both of these disks yields a new sutured manifold $N_{0}$, with the same set of sutures, whose new positive and negative boundaries are annuli. $\partial N_{0}$ is therefore a torus, and so, since $N_{0}$ is contained in $S^{3}$ and has connected boundary, $N_{0}$ is irreducible; a reducing 2 -sphere must separate boundary components. But $\partial N_{0}$ is still compressible; there is a disk $D$ whose boundary meets each suture in a single essential arc (Figure 5). Therefore, $N_{0}$ is a solid torus. In particular, because $D$ hits each suture exactly once, $N_{0}$ is a product sutured manifold (annulus) $\times I$. Therefore, $N$ is this product sutured manifold with two 1-handles attached, one to the positive boundary and one to the negative boundary. Consequently,

$M_{B} \cong M_{F} \cup N \cong M_{F} \cup(($ annulus $) \times I \cup(2$ one-handles $)) \cong M_{F} \cup(2$ one-handles $)$

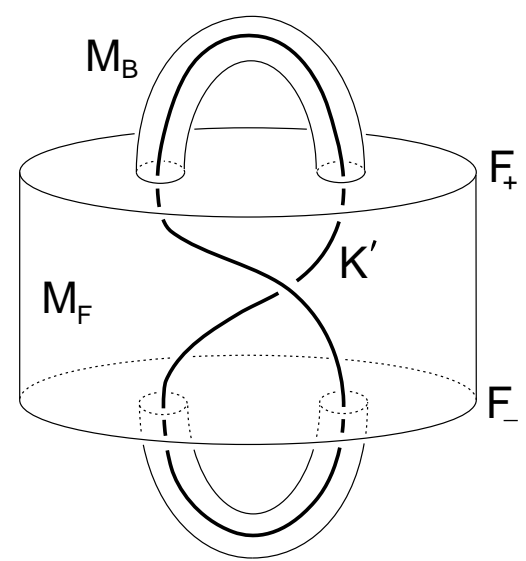

Figure 7

Therefore, $M_{B}=M_{F}$ with two (3-dimensional) one-handles attached, one having both ends on $F_{+}$, the other having both ends on $F_{-} . \partial_{h} \mathrm{~N}(\mathrm{~B})$ then consists of the surfaces $F_{+}$and $F_{-}$, each with a tube attached (increasing their genera by one). It has two obvious compressing disks, namely, the meridian disks of the two 1-handles. So $B_{F}$ is not essential in $S^{3}$. To kill these compressing disks, we will remove a knot $K^{\prime}$ from $M_{B}$ which intersects each of these disks exactly once (Figure 7 ). In other words, we will think of $B_{F}$ instead as a branched surface in $S^{3} \backslash \operatorname{int} N\left(K^{\prime}\right)=M_{K^{\prime}}$. But now we shall easily see that if $F$ is an incompressible Seifert surface for $K$, then $B_{F}$ is an essential branched surface in $M_{K^{\prime}}$, and remains essential after every non-trivial Dehn filling along $K^{\prime}$. In other words, $B_{F}$ is persistent for $K^{\prime}$.

Proposition. The branched surface $B_{F}$ is essential in the complement of any knot $K^{\prime}$ build as above.

Most of the arguments are identical to the proofs from [Br1]. To show that $B_{F}$ is essential in $M_{K^{\prime}}$, we need to know six things: 
(1) $B_{F}$ carries a lamination with full support.

This follows, as in [Br1] or [Ro], because the branch curve of $B_{F}$ does not intersect itself.

(2) $B_{F}$ does not carry a 2 -sphere, and $B$ has no disks of contact.

This is because, as in [Br1], $B_{F}$ has only one sector; $B_{F} \backslash \gamma$ is connected. Consequently, the branch equations [Oe2] for any surface carried by $B_{F}$, or surface of contact for $B_{F}$, will be $a=a+a$ or $a=a+a+1$, which have no positive solutions. In particular, $B_{F}$ carries no closed surfaces.

(3) $B_{F}$ does not carry a compressible torus.

This follows for the same reason as (2).

(4) $M_{0}=M_{K^{\prime}} \backslash \operatorname{int}\left(N\left(B_{F}\right)\right)$ does not have any monogons.

This follows from the fact that $B_{F}$ is transversely orientable; the boundary of a monogon traces out a transverse orientation reversing loop.

(5) $M_{0}$ is irreducible.

This is because $\partial\left(S^{3} \backslash \operatorname{int}\left(N\left(B_{F}\right)\right)\right)=\partial N\left(B_{F}\right)$ is connected, so $S^{3} \backslash \operatorname{int}\left(N\left(B_{F}\right)\right)$ is irreducible. Therefore, any reducing 2-sphere in $M_{0} \subseteq S^{3} \backslash \operatorname{int}\left(N\left(B_{F}\right)\right)$ bounds a 3-ball $B^{3}$ in $S^{3} \backslash \operatorname{int}\left(N\left(B_{F}\right)\right.$ ), which therefore contains $K^{\prime}$. But then $K^{\prime}$ would be null-homotopic in $M_{0}$, which is impossible since it has non-zero intersection number with each of the two compressing disks $D_{1}, D_{2}$.
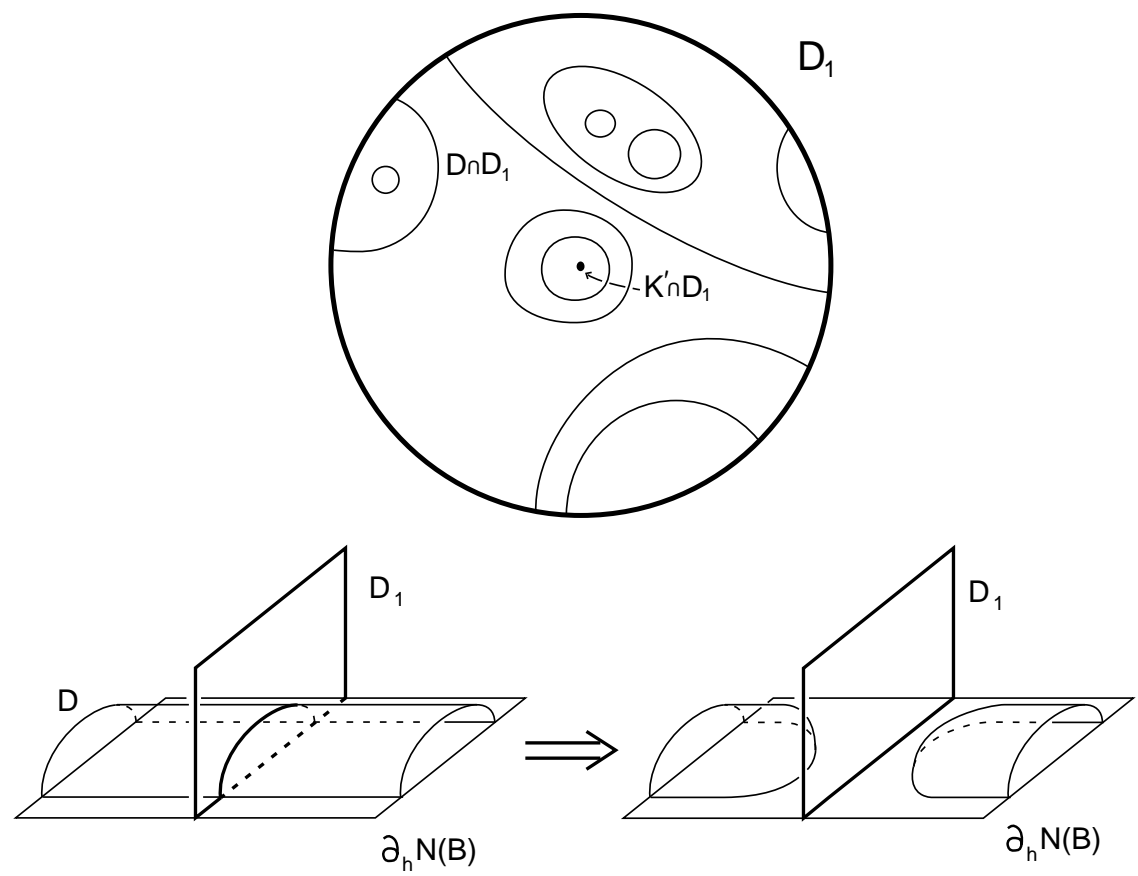

Figure 8

Figure 9

(6) The horizontal boundary $\partial_{h} N\left(B_{F}\right)$ of $B_{F}$ is incompressible in $M_{0}$.

Suppose $D$ is a compressing disk for $\partial_{h} N\left(B_{F}\right)$ in $M_{0}$; in particular, $D \cap K^{\prime}=\emptyset$. Consider $D \cap D_{1}$. This intersection consists of a finite number of circles and arcs. 
The circles come in two types; those which cut off a disk in $D_{1}$ which contains the intersection of $K^{\prime}$ with $D_{1}$, and those which don't (see Figure 8). We can remove the circles whose disks don't meet $K^{\prime}$ by a standard disk-swapping argument. The arcs of intersection can be removed by choosing an outermost arc, and using the disk it cuts off from $D_{1}$ to surger $D$ into two disks (see Figure 9). At least one of these is still a compressing disk for $\partial_{h} N\left(B_{F}\right)$. That disk has fewer arcs of intersection with $D_{1}$, so continuing with that disk will eventually lead to one with no $\operatorname{arcs}$.

This leaves the circles of intersection which surround the point $K^{\prime} \cap D_{1}$. But the innermost such circle cuts off a disk in $D$, which, together with the disk in $D_{1}$ it cuts off, produces a 2 -sphere which intersects $K^{\prime}$ exactly once. This is impossible, however, in $S^{3}$.

Therefore, we may assume that $D$ misses $D_{1}$, and therefore, by symmetry, that it misses $D_{2}$, as well. This means, then that we can push $D$ out of the two onehandles that were glued onto $M_{F}$ to create $M_{B}$. In particular, we may think of $\partial D$ as living in $F_{+}$or $F_{-}$(say, $\left.F_{+}\right)$. But since $F_{+}$is incompressible in $M_{F}, \partial D$ bounds a disk $D^{\prime}$ in $F_{+}$. In particular, $D \cup D^{\prime}$ bounds a 3-ball in $M_{F}$ (since $M_{F}$ is irreducible), so $D$ separates $M_{F}$. If $D^{\prime}$ misses the ends of the one-handle that we glued to $F_{+}$, then $D^{\prime}$ is contained in $\partial_{h} N\left(B_{F}\right)$, a contradiction. One the other hand, if $D^{\prime}$ does contain one or both of these disks, then $D$ separates one or both of these disks from both of the disks where the other one-handle was glued to $F_{-}$ (see Figure 10). But $K^{\prime}$ contains a pair of arcs running from the disks on $F_{+}$to the disks on $F_{-}$; if they ran from $F_{+}$to $F_{+}$, and from $F_{-}$to $F_{-}$, then $K^{\prime}$ would be a link instead of a knot. So at least one of these arcs must intersect $D$, since $D$ is separating. But this is also a contradiction.

Therefore, $B_{F}$ is an essential branched surface in $M_{K^{\prime}}$.

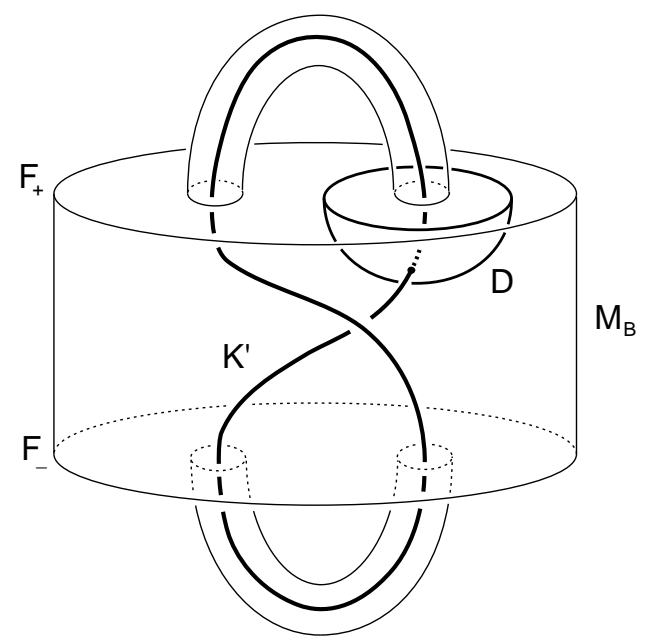

Figure 10

Persistence

The two disks $D_{1}$ and $D_{2}$ also allow us to show that $B_{F}$ remains essential after every non-trivial Dehn surgery along $K^{\prime}$. The only properties which do not immediately hold after Dehn surgery are (5) and (6), because (for (4)) $B_{F}$ re- 
mains transversely orientable, and for all of the other properties, $N\left(B_{F}\right)$ has not changed, only where it is embedded has, and so our previous proofs go through without change. Because $\partial D_{1}$ and $\partial D_{2}$ are not isotopic in $\partial_{h} N\left(B_{F}\right)$ (they live on distinct components), an argument due to Menasco [Me] shows that $\partial_{h} N\left(B_{F}\right)$ remains incompressible afrer non-trivial Dehn surgery along $K^{\prime}$. It remains to show that $M_{K^{\prime}}(p / q) \backslash \operatorname{int}\left(N\left(B_{F}\right)\right)=M_{(p, q)}$ is irreducible for all $p / q \neq 1 / 0$. Again, this argument is essentially the same as that given in [Br1].

Suppose $S$ is a reducing 2-sphere for $M_{(p, q)}$. We may assume that $S$ intersects (transversely) the core $\gamma$ of the solid torus glued onto $M_{K^{\prime}}$, in the fewest number of points. It is then standard that $S \backslash \operatorname{int}(N(\gamma))=S^{\prime}$ is an incompressible and $\partial$-incompressible surface in $M_{(p, q)} \backslash \operatorname{int} N(\gamma)=M_{K^{\prime}} \backslash \operatorname{int} N\left(B_{F}\right)=M_{2}$. The curves $S^{\prime} \cap \partial N(\gamma)$ are parallel curves of slope $p / q$ on $\partial N\left(K^{\prime}\right) \subseteq \partial M_{2}$.

The disks $D_{1}, D_{2}$ meet $M_{2}$ in annuli $A_{1}, A_{2}$. Consider $S^{\prime} \cap A_{i}$; it consists of circles and arcs. Trivial circles of intersection can be removed by isotopy. The arcs of intersection cannot meet the boundary component of $A_{i}$ coming from $\partial_{h} N\left(B_{F}\right)$, since $S$ misses $\partial M_{(p, q)}$. These arcs of intersection are therefore boundary parallel in $A_{i}$, and so can also be removed by isotopy, since $S^{\prime}$ is $\partial$-incompressible. After these isotopies, $S^{\prime}$ misses the boundary of $A_{i}$. If $\partial S^{\prime} \neq \emptyset$, then $\partial S^{\prime} \subseteq \partial N\left(K^{\prime}\right)$ misses a loop in $\partial N(\gamma)$ which represents a meridian of $K^{\prime}$, namely $A_{i} \cap \partial N(\gamma)$, and hence consists of meridional loops. So $p / q=1 / 0$, a contradiction.

Therefore $\partial S^{\prime}=\emptyset$, in particular, $S^{\prime}=S \subseteq M_{2} \subseteq M_{B}$. But since $M_{B}$ is irreducible, $S$ bounds a 3 -ball in $M_{B}$. This 3-ball must intersect, hence contain, $K^{\prime}$, since otherwise it is a 3 -ball in $M_{(p, q)}$. But this implies that $K^{\prime}$ is null-homotopic in $M_{B}$, which is impossible since it intersects the disk $D_{1}$ exactly once. So the reducing sphere $S$ cannot exist; $M_{(p, q)}$ is irreducible.

$\S 4$

Building The KNOT $K^{\prime}$, Without BUILDing $B_{F}$
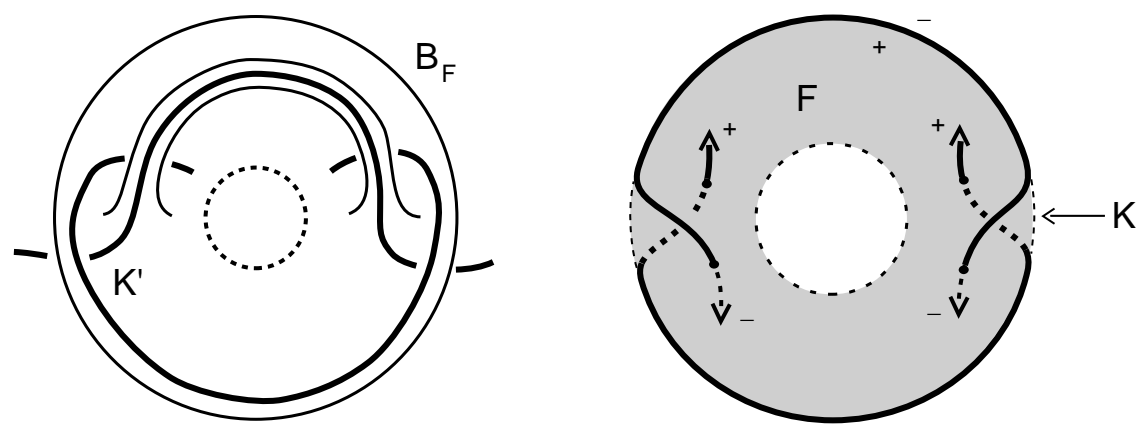

Figure 11

The knots $K^{\prime}$ obtained by this construction can be readily visualized directly from a picture of the incompressible Seifert surface $F$ of a knot $K$, without constructing $B_{F}$. The knots $K^{\prime}$ consist of two arcs running through the 1-handles of $S^{3} \backslash N\left(B_{F}\right)$, together with two arcs in $M_{F}$ running between these disks on $F_{+}$and

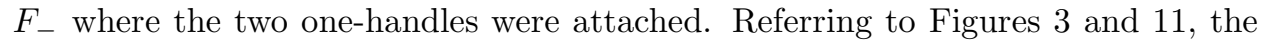
two arcs in the one-handle can be thought of as a pair of subarcs parallel to arcs of $K$, which then pass over and under one another at their ends. The ends then 
reverse direction and pierce the Seifert surface $F$ in four points (this reversal is required in order to be consistent with the sides of $F$ which the two 1-handles were attached to). These two arcs are then completed to a knot in any way that misses $F$ (and, technically, neighborhoods of the two added crossings). The easiest way to determine which way the two original arcs cross each other is to assign a ' + ' and '-' side to $F$, and think of one arc remaining slightly on the '-' side of $F$, and the other remaining slightly on the ' + ' side of $F$. This gives the correct 'parity' to the two crossings. Said slightly differently, we must make sure that both ends of one arc pierce $F$ from the same side, the other ends do so from the opposite side, and arrange the added two crossings accordingly.

The ends of these arcs coming from $K$ can then be assigned ' + 's and '-'s, marking which side of $F$ they are emerging from; in order to obtain a knot $K^{\prime}$, one then simply needs to join the four ends by arcs missing $F$, so that the + 's are joined to the -'s; see Figure 11.

\section{$\S 5$}

\section{SOME EXAMPLES}

The above construction can be carried out for any incompressible Seifert surface for a knot $K$. In the case that $K$ is the unknot, it is easy to see that all of the knots $K^{\prime}$ that one can build can be formed by tangle sum with a certain algebraic tangle; see [Br1]. For more complicated knots, the Seifert surfaces will have non-trivial topology, and so their complements will, as well. We can take advantage of this extra topology to build a much wider variety of persistently laminar knots $K^{\prime}$.
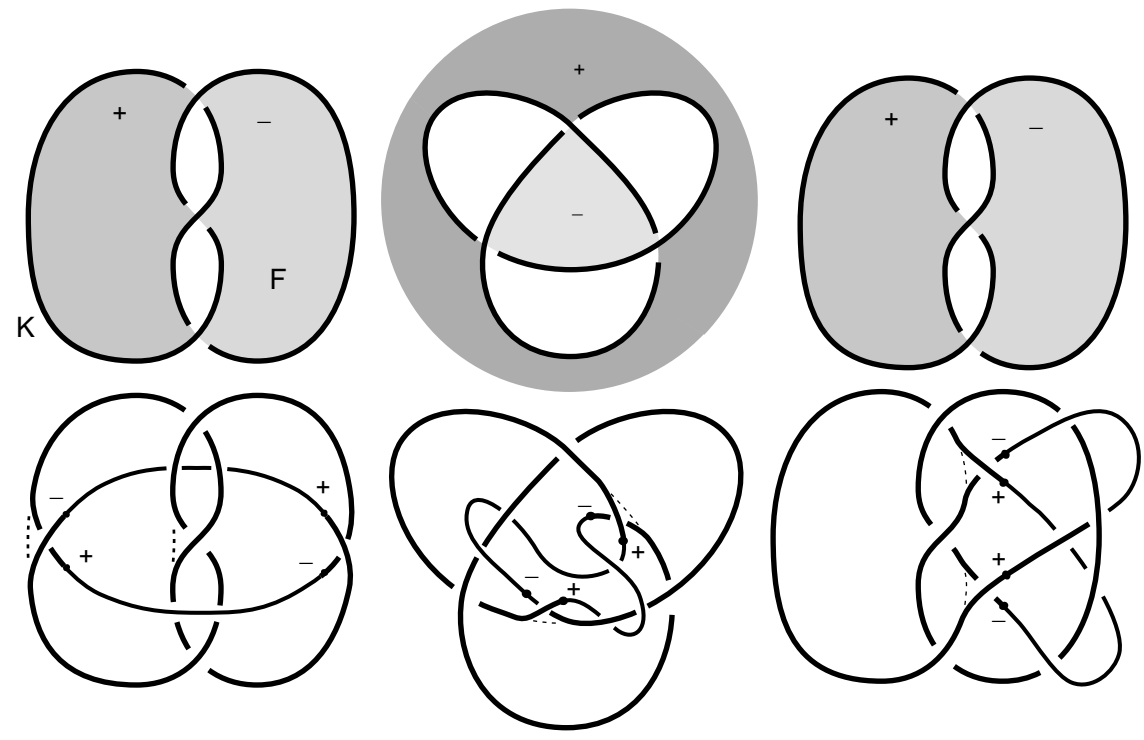

Figure 12

There are (at least) three ways to obtain an incompressible Seifert surface for a knot $K$ :

(1) run Seifert's algorithm on an alternating projection for an alternating knot [Cr];

(2) find a Seifert surface whose genus $=$ (span of the Alexander polynomial for the knot $) / 2[\mathrm{~S}]$ - in general, genus $(\mathrm{K}) \geq(\operatorname{span}) / 2$; 
(3) build a once-punctured torus whose boundary you know (for other reasons) is a non-trivial knot.

Note: all of these in fact give least genus Seifert surfaces.

If you have a surface which you suspect is least genus (hence incompressible), Gabai's theory of sutured manifold heirarchies [Ga1] can, in principle, prove it is. If you have a surface which you suspect is incompressible, Haken's normal surface theory (see [JO]) can, in principle, prove or disprove it.

We now illustrate this technique for building persistently laminar knots with a few examples.

Figure 12 shows several knots that can be built from the Seifert surface for the trefoil knot. Since the trefoil knot is fibered, all of its incompressible Seifert surfaces are isotopic [Th]. The middle picture shows, however, that different pictures of what are really the same surface can be helpful in this construction. The knots built here turn out to be, from left to right, $9_{46}, 10_{163}$, and $8_{9}$.

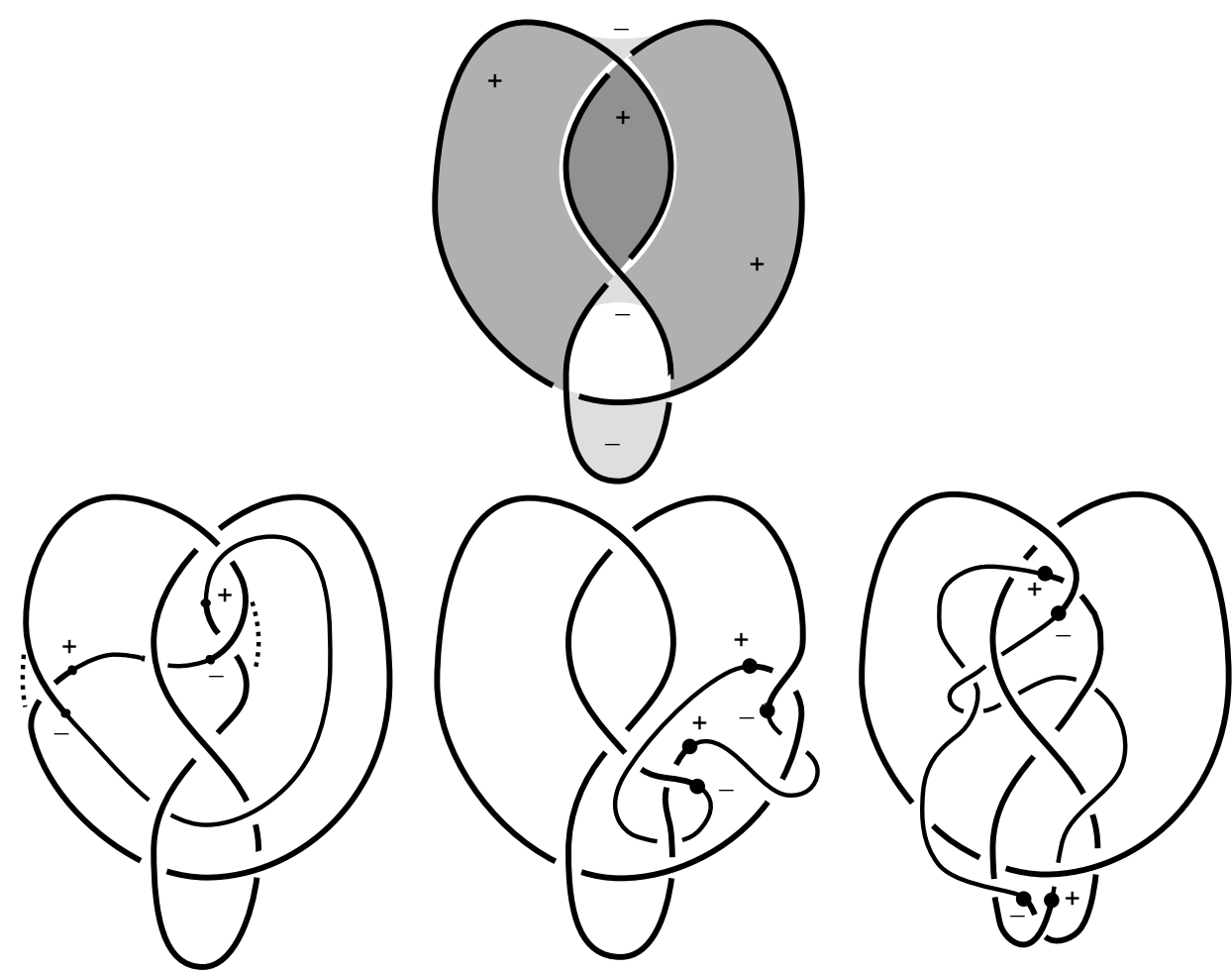

Figure 13

In Figure 13 we build some examples from the figure- 8 knot. The knots we build are, from left to right, $9_{44}, 10_{67}$, and $10_{146}$.

Finally, we show how to build an infinite family of knots, by using the same, localized, construction. Figure 14 shows how we can alter a knot in the neighborhood of one of its crossings, if our incompressible Seifert surface appears as in Figure 14a at the crossing. For example, applying this construction to the standard Seifert surface for a $(2,2 n+1)$ torus knot will build the twist knot with $2 n+6$ crossings, i.e., the 2-bridge knots with continued fraction expansion $[2,2 m]$ for $m \geq 3$. 


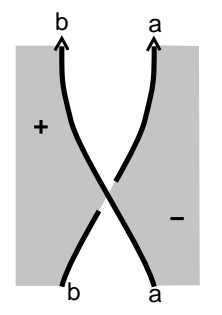

(A)

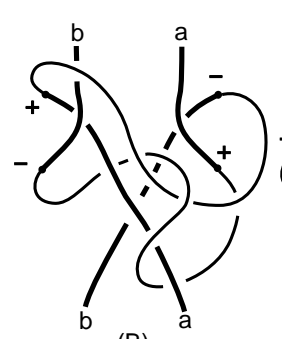

(B)

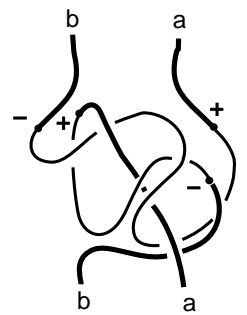

(D)

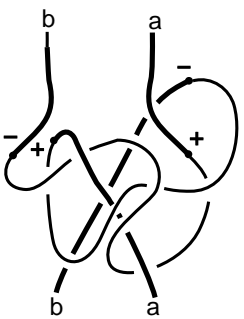

(C)

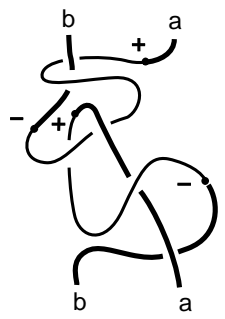

(E)

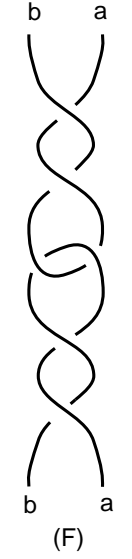

Figure 14

More generally, we can apply this construction to any $(2, \mathrm{n})$ cable of a knot $K$, since it is not hard to see that, starting with an incompressible Seifert surface $F$ for $K$, we can stitch together two parallel copies of $F$, as in Figure 15, to build an incompressible Seifert surface $F^{\prime}$ for the cable. The proof that the resulting surface is incompressible comes from the fact that the 'holes' in our picture can be spanned by product disks, in the terminology of sutured manifold theory; any compressing disk $D$ for $F^{\prime}$ meets these product disks in circles and arcs. The circles can be removed by disk-swapping. (Outermost) arcs, both of whose endpoints are on the same side of the product disk, can be removed by surgering $D$ along the subdisk each cuts off; one of the two resulting disks must still be a compressing disk for $F^{\prime}$. Finally, arcs running from top to bottom cannot exist, since the top and bottom edges of the product disk are on different sides of $F^{\prime}$. Once $D$ misses the product disks, its boundary lives on one of the copies of $F$ that we stitched together; the incompressibility of $F$ then implies that $\partial D$ bounds a disk in $F$, hence in $F^{\prime}$, a contradiction.
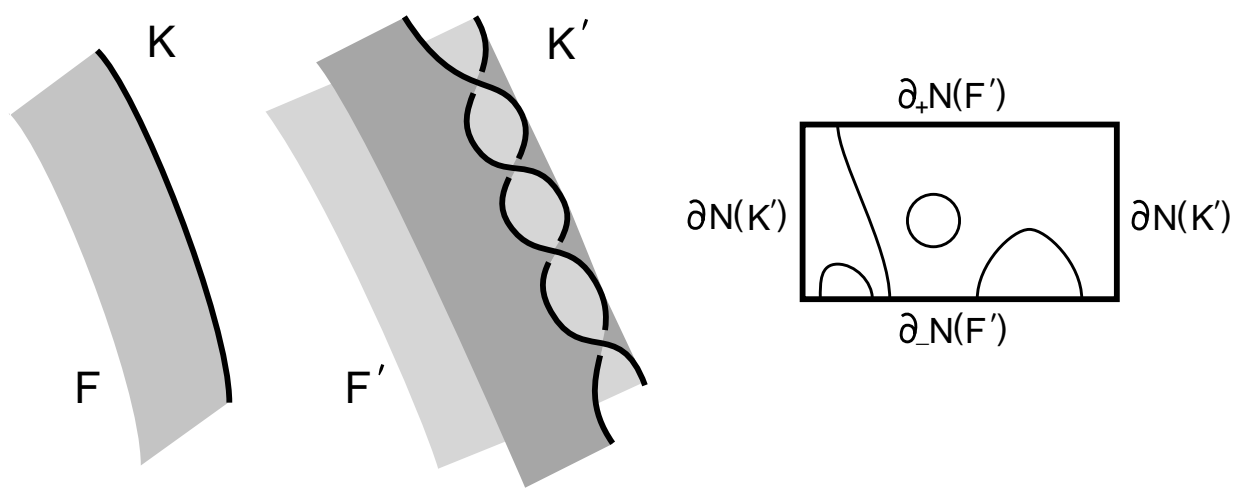

Figure 15 
This Seifert surface $F^{\prime}$ exhibits crossings that we can alter as in Figure 14; the resulting knot is a twisted Whitehead double of our original knot $K$. Applying this construction to cables with varying values of $n$, including negative ones (which yield the mirror image of Figure 14), yields persistent laminations for slightly less than half of all twisted doubles of $K$. Our twist knots above result from applying this construction to a 2-disk spanning the unknot.

Such constructions as above can be carried out in a literally unlimited number of ways. From the point of view of [Br1], for example, we can build a wealth of persistently laminar tangles by removing any ball in the complement of $B_{F} \cup D_{1} \cup D_{2}$ meeting our added arcs in a pair of arcs.

In particular, we can continue to add to the list of knots which are known to have strong Property P. The table below lists the knots in the standard knot tables which have so far been shown to have persistent laminations arising from this construction. A zip file containing illustrative projections of these knots, in a form readable by SnapPea $[\mathrm{We}]$, can be found at the following web page:

http://www.math.unt.edu/ britten/ldt/knots/knotlst1.html

\begin{tabular}{|c|c|c|c|c|c|c|c|c|c|}
\hline & & & & $6_{1}$ & & & & & \\
\hline $8_{3}$ & & & $\mathbf{8}_{6}$ & $8_{7}$ & $8_{8}$ & 8 & $\begin{array}{l}\mathbf{8}_{1} \\
\mathbf{8}_{10}\end{array}$ & $8_{11}$ & \\
\hline $8_{13}$ & $8_{14}$ & & $8_{16}$ & & & & $8_{20}$ & & \\
\hline & & & & & & $\mathbf{9}_{7}$ & $\mathbf{9}_{8}$ & & \\
\hline & $\mathbf{9}_{12}$ & & $\mathbf{9}_{14}$ & & & & & $\mathbf{9}_{19}$ & \\
\hline $\mathbf{9}_{21}$ & & & $\mathbf{9}_{24}$ & $\mathbf{9}_{25}$ & $\mathbf{9}_{26}$ & $\mathbf{9}_{27}$ & & & \\
\hline & $\mathbf{9}_{32}$ & & & & $\mathbf{9}_{36}$ & $\mathbf{9}_{37}$ & & $\mathbf{9}_{39}$ & \\
\hline & $\mathbf{9}_{42}$ & $\mathbf{9}_{43}$ & $\mathbf{9}_{44}$ & & $\mathbf{9}_{46}$ & $\mathbf{9}_{47}$ & $\mathbf{9}_{48}$ & & \\
\hline $10_{1}$ & & $10_{3}$ & $10_{4}$ & $10_{5}$ & $10_{6}$ & $10_{7}$ & & $10_{9}$ & $\mathbf{1 0}_{10}$ \\
\hline $\mathbf{1 0}_{11}$ & $10_{12}$ & $10_{13}$ & & & $\mathbf{1 0}_{16}$ & $\mathbf{1 0}_{17}$ & $\mathbf{1 0}_{18}$ & & $\mathbf{1 0}_{20}$ \\
\hline $10_{21}$ & $10_{22}$ & $10_{23}$ & $10_{24}$ & & $10_{26}$ & $10_{27}$ & & $10_{29}$ & $10_{30}$ \\
\hline & & & & $10_{35}$ & $10_{36}$ & & $10_{38}$ & & \\
\hline & & $10_{43}$ & & & & $10_{47}$ & $10_{48}$ & & $10_{50}$ \\
\hline & & $10_{53}$ & & $10_{55}$ & & & $1_{58}$ & & \\
\hline & $\mathbf{1 0}_{62}$ & $10_{63}$ & $\mathbf{1 0}_{64}$ & $10_{65}$ & & $\mathbf{1 0}_{67}$ & $\mathbf{1 0}_{68}$ & & \\
\hline & & & $\mathbf{1 0}_{74}$ & & & $\mathbf{1 0}_{77}$ & & $\mathbf{1 0}_{79}$ & $10_{80}$ \\
\hline & $10_{82}$ & $10_{83}$ & & & $10_{86}$ & $10_{87}$ & & & $\mathbf{1 0}_{90}$ \\
\hline $\mathbf{1 0}_{91}$ & & & $10_{94}$ & & & & $10_{98}$ & $\mathbf{1 0}_{99}$ & \\
\hline & $10_{102}$ & & $\mathbf{1 0}_{104}$ & & $\mathbf{1 0}_{106}$ & & & $10_{109}$ & $\mathbf{1 0}_{110}$ \\
\hline $\mathbf{1 0}_{111}$ & & & & & & & & & \\
\hline & $\mathbf{1 0}_{122}$ & & & $\mathbf{1 0}_{125}$ & $\mathbf{1 0}_{126}$ & $10_{127}$ & & & \\
\hline & & $10_{133}$ & $\mathbf{1 0}_{134}$ & & $\mathbf{1 0}_{136}$ & $\mathbf{1 0}_{137}$ & & & $\mathbf{1 0}_{140}$ \\
\hline $10_{141}$ & & $10_{143}$ & $10_{144}$ & & $\mathbf{1 0}_{146}$ & $10_{147}$ & $\mathbf{1 0}_{148}$ & & $\mathbf{1 0}_{150}$ \\
\hline & $\mathbf{1 0}_{152}$ & $10_{153}$ & $10_{154}$ & & & & $\mathbf{1 0}_{158}$ & $\mathbf{1 0}_{159}$ & \\
\hline & & $10_{163}$ & & $\mathbf{1 0}_{165}$ & & & & & \\
\hline
\end{tabular}

Combining these results with the results of the constructions listed in the introduction (see [Ga2]), the list of non-torus knots in the standard knot tables which are not yet known to have strong Property $\mathrm{P}$ becomes remarkably short; as of this writing, only the knots $10_{139}$, and $10_{161}=10_{162}$ (the Perko pair) remain. 
$\S 7$

\section{CONCLUding REMARKS}

It has long been conjectured that all non-trivial knots in $S^{3}$ have Property $\mathrm{P}$, that is, that non-trivial surgery on the knot can never yield a simply-connected manifold. On the other hand, not all knots have strong Property P: torus knots, for example, have surgeries with finite fundamental group, and cabled knots have reducible surgeries. Both of these properties preclude the surgery manifold from having universal cover $\mathbb{R}^{3}$.

The constructions we have described here represent only the simplest non-trivial sort of branched surface in $S^{3}$, yet they generate a wealth of examples of knots admitting persistent laminations, and hence having strong Property P. It is clear that much more can be gained, and learned, by applying this construction to other methods of building branched surfaces. This has, for example, been carried out by Hirasawa and Kobayashi [HK], for some other branched surface constructions.

One question that deserves an answer is whether or not every knot with strong Property $\mathrm{P}$ admits a persistent lamination. This is probably not the case, most likely because most exceptional Seifert-fibered spaces do not admit essential laminations $[\mathrm{Br} 3],[\mathrm{Cl}]$, although they do have universal cover $\mathbb{R}^{3}$. It is in fact not known that every non-torus alternating knot admits a persistent lamination, although as mentioned in the introduction, they do have strong Property P. There is at least one way to show that a knot does not admit a persistent lamination [BNR], but it requires knowing that one of the surgery manifolds is the 'right' kind of graph manifold. Whether or not this is the case for any alternating knot might be an interesting topic of study.

Another question which we can answer is whether or not, for all of the knots we can construct by this method, the surgery manifolds are in fact all Haken, i.e., contain an incompressible closed surface. This construction can easily generate a 2-bridge knot from certain other 2-bridge knots; for example, our last construction generated twist knots from 2-bridge torus knots. Since a 2-bridge knot exterior contains no closed, non- $\partial$-parallel incompressible surfaces [HT], it follows [Ha] that all but finitely many Dehn surgeries on these knots are non-Haken.

\section{Acknowledgements}

The author wishes to thank Rachel Roberts and Ramin Naimi for several helpful conversations during the preparation of this work.

\section{REFERENCES}

[Br1] M. Brittenham, Persistently laminar tangles, preprint.

[Br2] , in preparation.

[Br3] _ Essential laminations in Seifert-fibered spaces, Topology 32 (1993), 61-85.

[BNR] M. Brittenham, R. Naimi, and R. Roberts, Graph manifolds and taut foliations, J. Diff. Geom. 45 (1997), 446-470.

[Cl] E. Claus, Essential laminations in closed Seifert-fibered spaces, Thesis, Univ. of Texas at Austin (1991).

[Cr] R.H. Crowell, Genus of alternating link types, Annals of Math. 69 (1959), 258-275.

[De] C. Delman, Essential laminations and Dehn surgery on 2-bridge knots, Topology Appl. 63 (1995), 201-221.

[DR] C. Delman and R. Roberts, Constructing essential laminations in alternating knot exteriors, to appear.

[Ga1] D. Gabai, Genera of the arborescent links, Memoirs of the AMS 339 (1986), 1-98. 
[Ga2] , Problems in foliations and laminations, Geometric topology (Athens, GA, 1993), Studies in Advanced Mathematics, vol. 2, Amer. Math. Soc., Providence, RI, 1997, pp. 133.

[GO] D. Gabai and U. Oertel, Essential laminations in 3-manifolds, Annals of Math. 130 (1989), 41-73.

[Ha] A. Hatcher, On the boundary curves of incompressible surfaces, Pacific J. Math. 99 (1982), 373-377.

[HT] A. Hatcher and W. Thurston, Incompressible surfaces in 2-bridge knot complements, Inventiones Math. 79 (1985), 225-246.

[HK] M. Hirasawa and T. Kobayashi, Pre-taut sutured manifolds and essential laminations, in preparation.

[JO] W. Jaco and U. Oertel, An algorithm to decide if a 3-manifold is a Haken manifold, Topology 23 (1984), 195-209.

[Me] W. Menasco, Closed incompressible surfaces in alternating knot and link complements, Topology 23 (1984), 37-44.

[Na1] R. Naimi, Essential laminations in 3-manifolds obtained by surgery on 2-bridge knots, Contemp. Math. 164 (1994), 183-186.

[Na2] Constructing essential laminations in 2-bridge knot surgered 3-manifolds, Pacific J. Math. 180 (1997), 153-186.

[Oe1] U. Oertel, Affine laminations and their stretch factors, Pacific J. Math. 182 (1998), 303328.

[Oe2] U. Oertel, Incompressible branched surfaces, Inventiones Math. 76 (1984), 385-410.

[R] D. Rolfsen, Knots and Links, Publish or Perish Press, 1976.

[Ro] R. Roberts, Constructing taut foliations, Comment. Math. Helv. 70 (1995), 516-545.

[S] H. Seifert, Über das Geschlecht von Knoten, Math. Annalen 110 (1934), 571-592.

[Th] W. Thurston, A norm for the homology of 3-manifolds, Memoirs of the AMS 59339 (1986), 99-130.

[We] J. Weeks, SnapPea, a program for creating and studying hyperbolic 3-manifolds, available for download from www.geom.umn.edu.

[Wu] Y.-Q. Wu, Dehn surgery on arborescent knots, J. Diff. Geom. 43 (1996), 171-195.

Department of Mathematics, Box 305118, University of North Texas, Denton, TX 76203

E-mail address: britten@unt.edu 